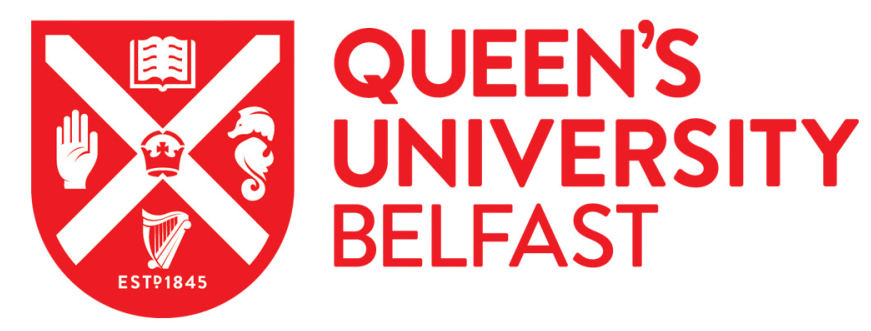

\title{
Atomic-scale imaging of few-layer black phosphorus and its reconstructed edge
}

Lee, Y., Yoon, J-Y., Scullion, D., Jang, J., Santos, E. J. G., Jeong, HY., \& Kim, K. (2017). Atomic-scale imaging of few-layer black phosphorus and its reconstructed edge. Journal of Physics D: Applied Physics, 50(8). https://doi.org/10.1088/1361-6463/aa5583

Published in:

Journal of Physics D: Applied Physics

Document Version:

Peer reviewed version

Queen's University Belfast - Research Portal:

Link to publication record in Queen's University Belfast Research Portal

Publisher rights

(c) 2017 IOP Publishing Ltd.

This work is made available online in accordance with the publisher's policies. Please refer to any applicable terms of use of the publisher.

\section{General rights}

Copyright for the publications made accessible via the Queen's University Belfast Research Portal is retained by the author(s) and / or other copyright owners and it is a condition of accessing these publications that users recognise and abide by the legal requirements associated with these rights.

Take down policy

The Research Portal is Queen's institutional repository that provides access to Queen's research output. Every effort has been made to ensure that content in the Research Portal does not infringe any person's rights, or applicable UK laws. If you discover content in the Research Portal that you believe breaches copyright or violates any law, please contact openaccess@qub.ac.uk. 
Atomic-scale imaging of few-layer black phosphorus and its reconstructed edge

Yangjin Lee ${ }^{1}$, Jun-Yeong Yoon ${ }^{1}$, Declan Scullion ${ }^{3}$, Elton J. G. Santos ${ }^{3,4}$, Hu Young Jeong ${ }^{2, *}$, and Kwanpyo $\mathrm{Kim}^{1, *}$

${ }^{1}$ Department of Physics and Applied Mathematics, Ulsan National Institute of Science and Technology (UNIST), Ulsan 689-798, South Korea.

${ }^{2}$ UNIST Central Research Facilities (UCRF), Ulsan National Institute of Science and Technology (UNIST), Ulsan 689-798, South Korea.

${ }^{3}$ School of Mathematics and Physics, Queen's University Belfast, Belfast, BT95AG, United Kingdom.

${ }^{4}$ School of Chemistry and Chemical Engineering, Queen's University Belfast, Belfast, BT95AL, United Kingdom.

*Address correspondence to K.K. (kpkim@unist.ac.kr) or H.Y.J. (hulex@unist.ac.kr)

Black phosphorus (BP) has recently emerged as an alternative two-dimensional semiconductor owing to its fascinating electronic properties such as tunable band gap and high charge carrier mobility. The structural investigation of few-layer black phosphorus, such as identification of layer thickness and atomic-scale edge structure, are of great importance to fully understand its electronic and optical properties. Here we report atomic-scale analysis of few-layered BP performed by aberration corrected transmission electron microscopy (TEM). We establish the layer-number-dependent atomic resolution imaging of few-layer BP via TEM imaging and image simulations. We also find that the electron beam can be utilized to form BP nanoribbons with crystalline edge structures. Atomic resolution imaging of BP clearly shows the reconstructed edge structures, which is also corroborated by van der Waals first principles calculations on the edge stability. Our study on the precise identification of BP thickness and 


\section{atomic-resolution imaging of edge structures will lay the groundwork for investigation of few- layer BP, especially BP in nanostructured forms.}

A century after its discovery, ${ }^{1-3}$ black phosphorus (BP) has regained much attention as an alternative two-dimensional (2D) material owing to its promising electrical, optical and chemical properties. ${ }^{4-19}$ As a layered structure, BP has the largely tunable bandgap as a function of the number of layers $(0.35-2.0 \mathrm{eV})$, which can bridge the missing band-gap range from the currently available various 2D materials. ${ }^{9,15,16,20}$ BP also poses various interesting electrical, mechanical, and optical properties, such as large tunability by strain ${ }^{20-22}$ and high in-plane anisotropy. ${ }^{11,16,23-25}$ Moreover, researchers have recently demonstrated the high charge carrier mobility from few-layer $\mathrm{BP}^{5,6,8,15,16}$ opening up various interesting electronic applications ${ }^{5,26,27}$ and fundamental studies. ${ }^{10,11}$

Atomic-scale structural analysis of few-layer BP is essential to fully understand its electronics and optical properties. The various defects ${ }^{28,29}$ have a profound effect on charge carrier dynamics, which becomes more important for the few-layer form of BP. BP nanoribbons also have various interesting properties, including edge-type-dependent electronic properties and special edge states, as shown by recent theoretical studies. ${ }^{22,30-32}$ Until now, only a few experimental results on the structural characterization of BP using various microscopy techniques have been reported. ${ }^{9,33-35}$ Although these reports provide general structural analysis on BP, atomic-scale imaging and analysis of structural modification of BP are mainly unexplored at this point.

Here we report atomic-scale analysis of few-layered BP performed by aberration corrected transmission electron microscopy (TEM). We establish the layer-number-dependent atomic resolution imaging of few-layer BP via TEM experiments and image simulations. In addition, we find that the electron beam can be utilized to form BP nanoribbons with crystalline edge structures. 
TEM imaging reveals that the BP edge shows the reconstructed edge configuration, which is also confirmed by first principles calculations with van der Waals dispersion force method. Our study on the precise identification of BP thickness and atomic resolution imaging of BP edges will lay the groundwork for investigation of electrical and optical properties of BP nanostructures.

For TEM analysis, few-layer BP samples are prepared using the conventional mechanical exfoliation and transfer method. (See Method for details.) During the sample preparation process, we minimize sample's exposure time to ambient environment in order to reduce sample degradation. ${ }^{8,9,35}$ Optical characterizations before TEM analysis are not performed on samples because of possible photon-induced degradation. ${ }^{35}$ Figure 1a shows a low-magnification TEM image of a typical BP flake. Without tilting samples, BP usually exhibits the crystal direction viewed along zone axis [010] as shown in Fig. 1b and 1d. The Fourier transform of the image clearly shows the diffraction signal with lattice parameters which are consistent with previous results ${ }^{2}$ (Fig. 1e and Table S1). The figure 1f shows the enlarged view of the dashed box in Figure 1d, which agree well with the simulated TEM image (Fig. 1g) at reasonable simulation conditions, thickness and defocus value of $21 \mathrm{~nm}$ and $6 \mathrm{~nm}$

Some transferred BP flakes display folded edge structures and this allows us to observe BP at different crystallographic directions, even without tilting of samples. Figure 1h shows a TEM image around the flake edge where the crystal structure at zone axis [100] is clearly observed. At [100] zone axis, the puckered layered structure of BP can be clearly observed. The interlayer distance of BP is found to be $5.27 \AA$, which is consistent with previously reported results. ${ }^{2}$ The zoom-in image and simulated image are also in a good agreement with simulation conditions of thickness of $20 \mathrm{~nm}$ and defocus value of $7 \mathrm{~nm}$. With tilting of the specimens, atomic resolution imaging at extra zone axes is also performed as shown in Supplementary Figure S1. Especially, atomic resolution TEM images from [101] zone axis can differentiate $\mathrm{AB}$ and AA stacking and our observation indicate that bulk BP 
has mainly AB stacking (Supplementary Figure S1f-j). Our energy calculations for different stacking also confirm that AB stacking is the most stable stacking configuration (Supplementary Figure S2). We also find that all the lattice parameters and d-spacing values are consistent with previously reported values, ${ }^{2}$ which is summarized in Table S1.

The precise and facile identification of BP thickness is a prerequisite for investigation of various properties. Figure 2 summarizes how the number of BP layers can be identified through atomic resolution TEM imaging and simulations. Figure 2a shows the TEM image of much thinner (three to seven layers) BP specimen where the thickness is not homogeneous in the different locations due to electron-beam-induced sputtering (Supplementary Figure S3). The comparison of the intensity modulation and its pattern along c-axis of BP is performed between observed TEM images and simulation results. Since the phase contrast TEM image depends on the number of BP layers and defocus value, we perform a series of image simulations along [010] zone axis as a function of the layer number as well as the defocus value, where we assume the $A B$ stacking (Supplementary Figure S4). One thing to note is that even and odd layer numbers produce distinct image patterns. Simulations with even number of layers (for example, double layer) show the intensity modulation with the half of usual bulk lattice parameters due to the symmetric AB stacking. On the other hand, with an odd number of layers (monolayer and triple layer), the simulated images display the intensity modulation with the periodicity of usual lattice parameters of bulk BP.

We assign the layer numbers and defocus values for the observed TEM images by comparison with simulated images. For example, top image of Figure 2b, the zoom-in image of marked regions A in Figure 2a, shows the distinct image pattern which can originate from the negative defocus values around -6nm and odd number of layer. To assign the precise layer number, we compare the intensity line profiles along the crystal c-axis between the simulated and observed TEM images (Figure 2c). The experimentally observed and simulated TEM image intensity 
modulation differ by a factor of two, which is often called the Stobb's factor. ${ }^{36-38}$ After taking account of this factor, the simulation intensity pattern with 5 layers matches the observation. The TEM images obtained from other locations of the specimen also undergo a similar process and we assign the number of layers and defocus values (Figure 2d), which is also confirmed by the intensity modulation analysis (Supplementary Figure S5 and Table S2) along c-axis (Figure 2e). We find that the BP specimen imaged in Figure 2a has a thickness ranging from three to seven layers.

Now we start our discussion on the edge structure of BP. The investigation of edge structure of BP is an important topic as it significantly influences various physical properties of BP. There are a few recent studies on BP edges and nanoribbons, especially by theoretical calculations. ${ }^{30,31}$ However, there is still a limited number of experimental studies on BP edges. ${ }^{33}$ As shown in Supplementary Figure S4, as-prepared BP specimens exhibit amorphous edge structure, where the amorphous edge regions of several nanometers are always observed. This amorphous edge is possibly due to the degradation during the sample preparation process.

The edge structure of BP crystals can be structurally modified by e-beam irradiation during TEM imaging. ${ }^{39}$ Remarkably, the crystalline edge structure can be obtained via this method. Figure 3a and $3 \mathrm{~b}$ shows the changes of sample over 15-second e-beam exposure. The atoms at the amorphous edge (indicated by yellow arrows in Figure 3a) can be preferentially sputtered out, exposing the crystalline edge structure. The crystal direction of exposed edge shows zigzag (ZZ) edge direction. Figure 3c is the zoom-in image of BP edge where the periodic edge structure over five unit-cells is clearly observed. Moreover, the image pattern at the edge shows a higher intensity modulation compared to the basal plane. This strongly suggests that there is the reconstructed edge formation.

To have a better understanding on atomic-scale structure of ZZ edge, we calculate the relaxed edge structures with various possibilities using first principles calculations with van der Waals 
interactions (See Methods). We find that reconstructed ZZ edges (type 1 and type 2) exhibit similar edge formation energies compared to zigzag (ZZ) or armchair (AC) edge configurations (Supplementary Figure S6). To compare with experimental TEM images, a series of image simulations assuming different $\mathrm{ZZ}$ edge structures including usual $\mathrm{ZZ}$ edge termination (Supplementary Figure S8) and two types of reconstructed edge configurations are undertaken (Supplementary Figure S9 and S10). Since the observed area has three-layer thickness, which is determined by the previous image pattern and intensity modulation analysis, we focus on the simulated images from triple-layer. By comparison, we find that the observed ZZ edge is consistent with the type-2 ZZ edge reconstruction (Figure 3d). The usual ZZ edge without reconstruction (Figure 3g and 3h) and the type-1 reconstructed edge (figure $3 \mathrm{i}$ and $3 \mathrm{j}$ ) are not consistent with the observed image. We note that the observed type-2 reconstructed ZZ edge was theoretically studied together with some experimental evidence but the direct atomic resolution edge imaging was not previously performed. ${ }^{33}$

Finally, we discuss the sample thinning and BP nanoribbon formation induced by electron beam irradiation. Figures 4a-e show a time series of structural modification of BP under electron beam. The same series of images are overlaid with different colors in Figures 4f-j for easy identification of structural changes. Different colors indicates triple-layer region (blue), thicker area (pink) and amorphous regions (yellow). The sample thinning with electron-beam sputtering is observed from Figure $4 \mathrm{f}$ and $4 \mathrm{~g}$; the region overlaid with pink color (thicker area) is gradually replaced by the blue region (three layers).

Electron-beam is one of useful ways to manipulate the materials at nanoscale and we demonstrate that BP nanoribbons can be formed by prolonged e-beam exposure. Figure 4d and $4 \mathrm{i}$ clearly show that the formation of approximately 4nm-wide BP nanoribbons with crystalline edge. After prolonged e-beam irradiation, the BP nanoribbon is amorphized to form amorphous BP nano- 
constriction with a less than $2 \mathrm{~nm}$ neck width. Consequently, the nano-constriction breaks down (See Supporting Movie). The sputtering mechanisms during TEM imaging including calculations of knock-on damage threshold for phosphorene will be an important experimental issue and we are currently investigating this effect in detail.

In conclusion, the atomic-scale structure of few-layered BP and its reconstructed ZZ edges were investigated by Cs-corrected TEM and imaging simulation. The precise and facile characterization methods of BP thickness demonstrated in our study will lead to various fundamental studies, such as measurements of layer-number-dependent electrical and optical properties. We also demonstrate that electron beam irradiation can be used to form BP nanoribbons as well as to expose crystalline reconstructed ZZ edge. Further TEM analysis on BP is expected to shed light on various defect structures and structural degradation mechanisms. 


\section{Methods}

Black phosphorus sample preparation: Black phosphorus (BP) crystals were purchased from Smart Elements (purity, 99.998\%). To prepare TEM samples, BP flakes were exfoliated onto $\mathrm{SiO}_{2} / \mathrm{Si}$ wafers using conventional mechanical exfoliation method. Exfoliated BP flakes were transferred to Quantifoil Au TEM grids using direct transfer method. ${ }^{40}$ To thin BP flakes and remove surface residues, gentle plasma cleaning with $\mathrm{H}_{2}$ and $\mathrm{O}_{2}$ gas environment was carried out using plasma cleaner (Advanced plasma system, Gatan, USA) for 5 minutes with $10 \mathrm{~W}$ input power. To minimize the oxidation of BP specimens, BP samples were immediately loaded into TEM chamber after sample preparation process.

TEM characterizations and image simulations: The atomic resolution imaging of BP were performed with a FEI Titan G2 operated at $80 \mathrm{kV}$, which is equipped with image Cs aberration corrector and monochromator. For the TEM time series, the exposure time of 0.3 seconds together with the processing time of 0.25 seconds were used. This results in the image frame time of 0.55 second per image. All the TEM image simulations were performed using MacTempas software. The BP crystal structure of BP with $a=3.31 \AA, b=10.48 \AA$ and $c=4.37 \AA$ (space group $\mathrm{Cmca}^{41}$ ) were used. The b-axis is the layer stacking direction.

Theoretical calculations: Calculations were based on $a b$ initio density functional theory using the SIESTA method ${ }^{42}$ and the VASP code ${ }^{43,44}$. The generalized gradient approximation ${ }^{45}$ along with the optB88-vdW ${ }^{46,47}$ functional was used in both methods, together with a double- $\zeta$ polarized basis set in Siesta, and a well-converged plane-wave cutoff of $500 \mathrm{eV}$ in Vasp. Projected augmented wave method (PAW) $)^{48,49}$ for the latter, and norm-conserving (NC) Troullier-Martins pseudopotentials ${ }^{50}$ for the former, have been used in the description of the bonding environment for P. NC pseudopotentials include non-linear-core corrections (NLCC) ${ }^{51}$ to correctly account for the weak interactions between core and valence densities. The pseudocore radii $r_{N L C C}\left(a_{o}\right)$ (in Bohrs) together with the different $l$ channels $r_{l}\left(a_{o}\right)$ have been optimized and the values are: $r_{s}\left(a_{o}\right)=1.83, r_{p}\left(a_{o}\right)=1.83, r_{d}\left(a_{o}\right)=1.83$, $r_{f}\left(a_{o}\right)=1.83, r_{N L C C}\left(a_{o}\right)=1.45$. The shape of the NAOs was automatically determined by the algorithms described in ${ }^{42}$. The cutoff radii of the different orbitals were obtained using an energy shift of 50 $\mathrm{meV}$, which proved to be sufficiently accurate to describe the geometries and the energetics. Atomic coordinates were allowed to relax until the forces on the ions were less than $0.04 \mathrm{eV} / \AA$ under the conjugate gradient algorithm. Further relaxations $(0.01 \mathrm{eV} / \AA)$ do not change appreciably the energetics and geometries. The lattice constants for the monolayer unit cell were optimized and found to be $a=3.297 \AA, b=22.1220 \AA, c=4.655 \AA$ in Siesta which is in good agreement with the 
results obtained using Vasp, a=3.295 $\AA, b=22.1219 \AA$, c=4.535 $\AA$. To model the system studied in the experiments, we created large supercells containing up to 136 atoms to simulate the interface between different nanoribbon layers and edge reconstructions in the phosphorene. To avoid any interactions between supercells in the non-periodic direction, a $20 \AA$ vacuum space was used in all calculations. In addition to this, a cutoff energy of 100 Ry was used to resolve the real-space grid used to calculate the Hartree and exchange-correlation contribution to the total energy. For the phosphorene sheets, the Brillouin zone was sampled with a 10x8x1 grid under the Monkhorst-Pack scheme $^{52}$, which gives similar results as those using a finer $17 \times 15 \times 1 \mathrm{k}$-sampling. In addition to this we used a Fermi-Dirac distribution with an electronic temperature of $\mathrm{k}_{\mathrm{B}} \mathrm{T}=20 \mathrm{meV}$.

\section{Acknowledgements}

This work is supported by Basic Science Research Program through the National Research Foundation of Korea (NRF) funded by the Ministry of Education (NRF-2014R1A1A2058178). D.S. thanks the studentship from the EPSRC-DTP award. E.J.G.S. acknowledges the use of computational resources provided by the Atomistic Simulation Center, and the Extreme Science and Engineering Discovery Environment (XSEDE), supported by NSF grants number TG-DMR120049, TGDMR150017; as well as the Queen's Fellow award through the startup grant number M8407MPH. 


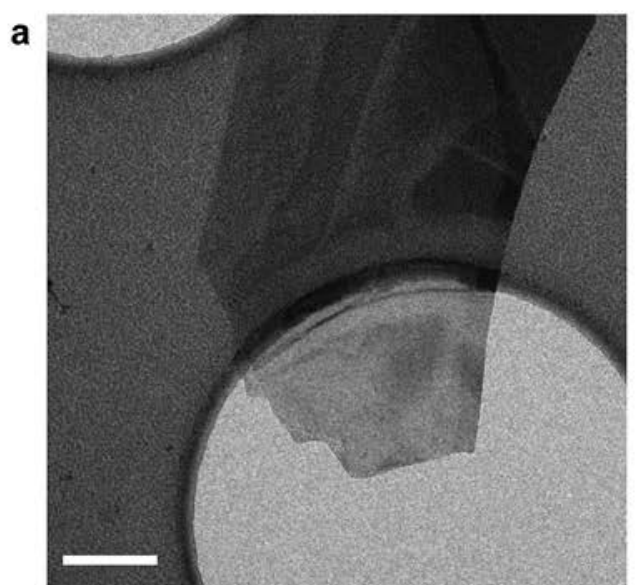

b

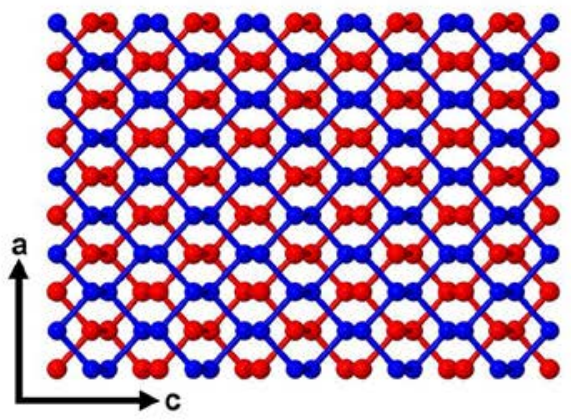

d
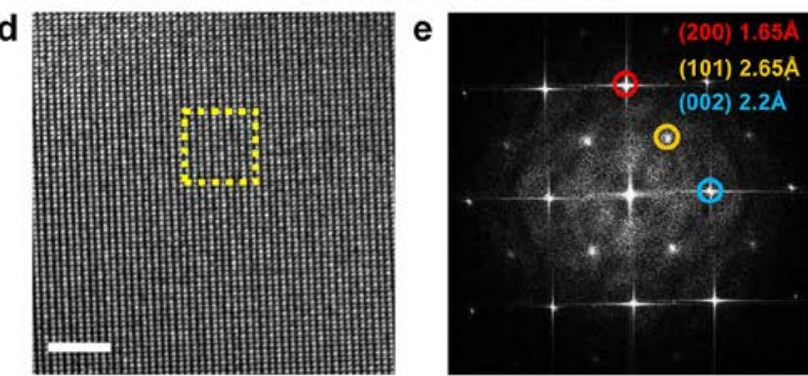

h

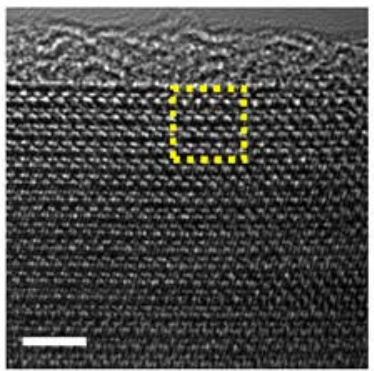

i

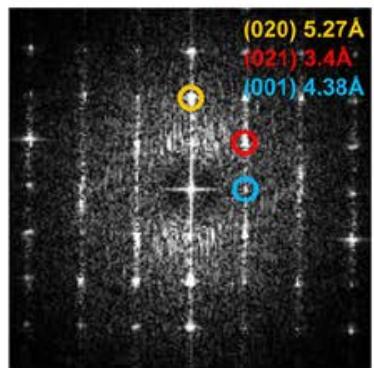

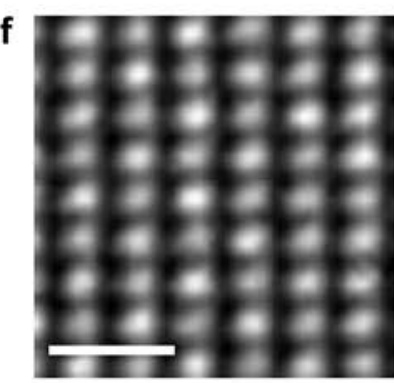

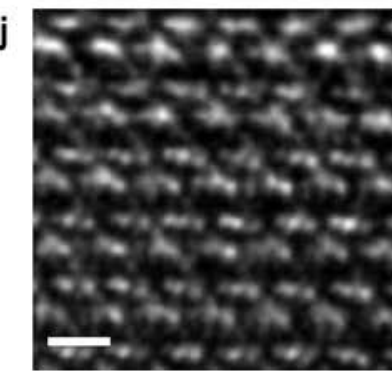

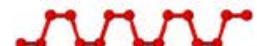

חนชน

กัน⿰冫欠

นดน
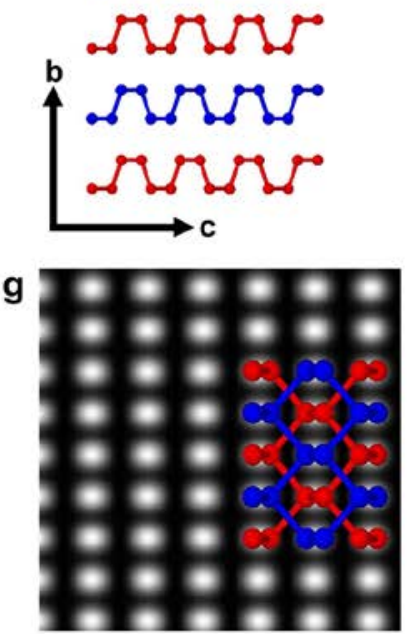

k

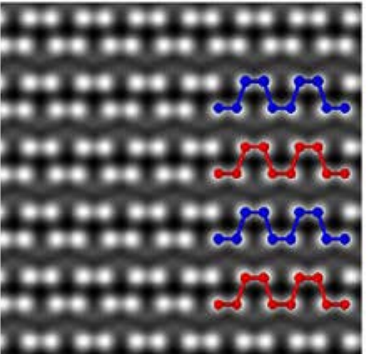

Figure 1. TEM analysis of black phosphorus (BP) at various crystal zone axes. (a) Low magnification TEM image of a BP sample. Scale bar, 500nm. (b,c) Atomic model of BP shown at [010] and [100] zone axes, respectively. (d) TEM image of the BP at [010] zone axis. Dashed box is the field of view for panel f. Scale bar, 2nm. (e) Fourier transform of panel d. The circles indicate low-index diffraction spots. (f) Enlarged view of the dashed box in panel d. Scale bar, 0.5nm. (g) TEM image simulation result from the crystal structure shown in panel b. The locations of phosphorus atoms are overlaid as circles. (h) TEM image of a BP flake at [100] zone axis. Scale bar, 2nm. (i) Fourier transform of panel h. The circles indicate low-index diffraction spots. (j) Enlarged view of box in panel h. Scale bar $0.5 \mathrm{~nm}$. (k) Simulated TEM image from the crystal structure shown in panel c. 

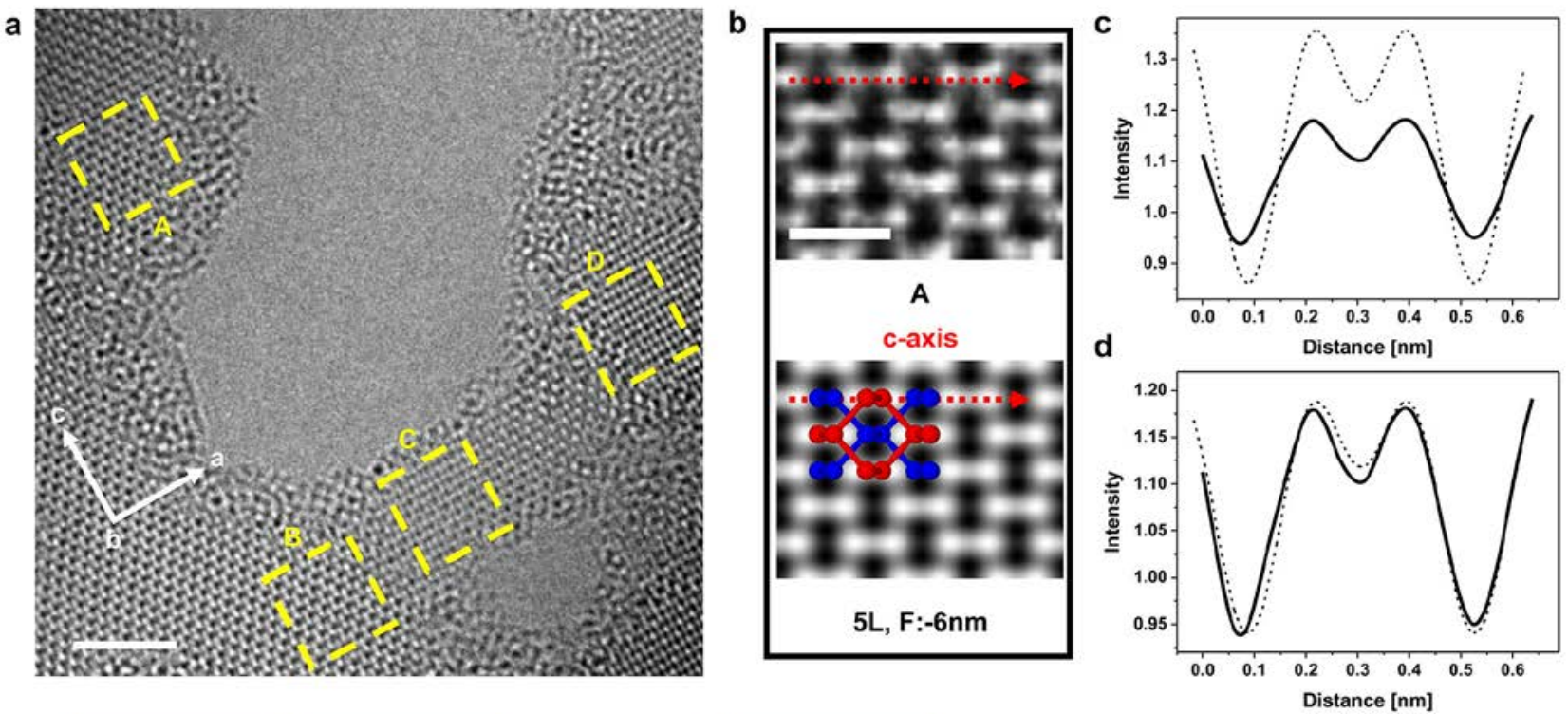

e
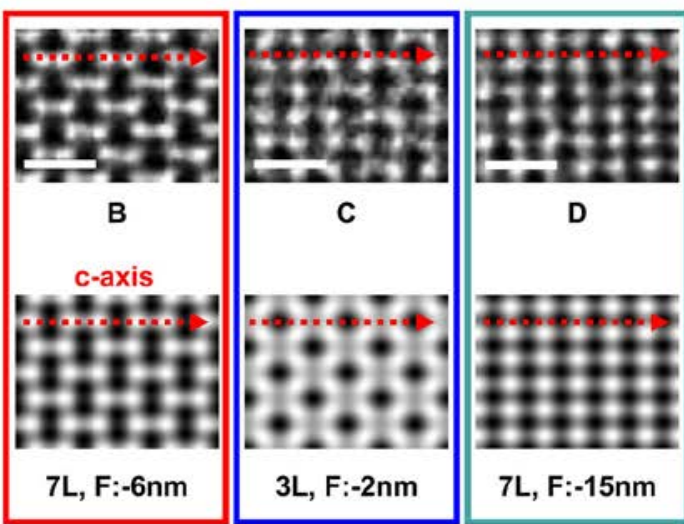

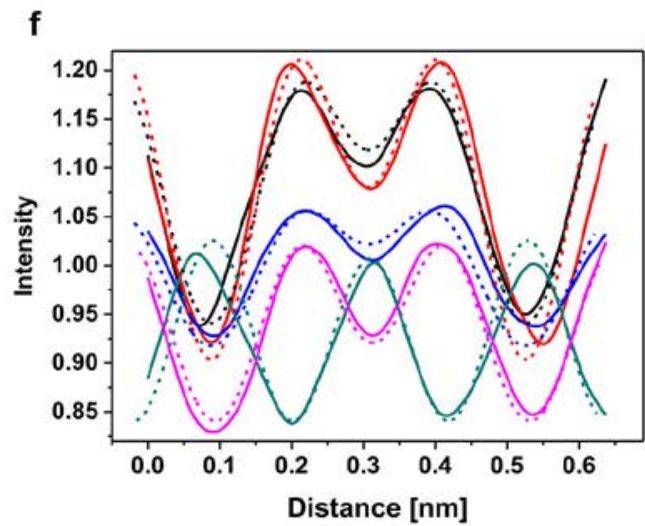

Figure 2. Determination of BP thickness by TEM imaging. (a) TEM image of BP showing inhomogeneous thickness at different locations. Scale bar, $2 \mathrm{~nm}$. The area inside dashed squares is used for intensity profile analysis. (b) Zoom-in image of marked region A and simulated image with 5 layer thickness and defocus value of - $6 \mathrm{~nm}$ (labeled as 5L -6f). The horizontal direction of images correspond to the c-axis of BP crystal. (c) Intensity profiles from marked region A (solid line) and simulated image (dashed line) along the c-axis (red dashed line in panel b). Experimental intensity profile is the average from 5 unit cells. (d) The same plot with normalized simulation profile. (e) TEM images (top) acquired at different sample locations and simulated images (bottom) from the chosen thickness and defocus values. Scale bar, $0.5 \mathrm{~nm}$. (f) Intensity profiles from TEM images (solid lines) and simulation images (dashed line) along the c-axis. Black, red, blue, cyan, and pink colors indicates the data from region A, B, C, D, and D'. All the simulation plots are normalized $(\times 0.5)$. All experimental intensity profiles are the average from 5 unit cells. 
a

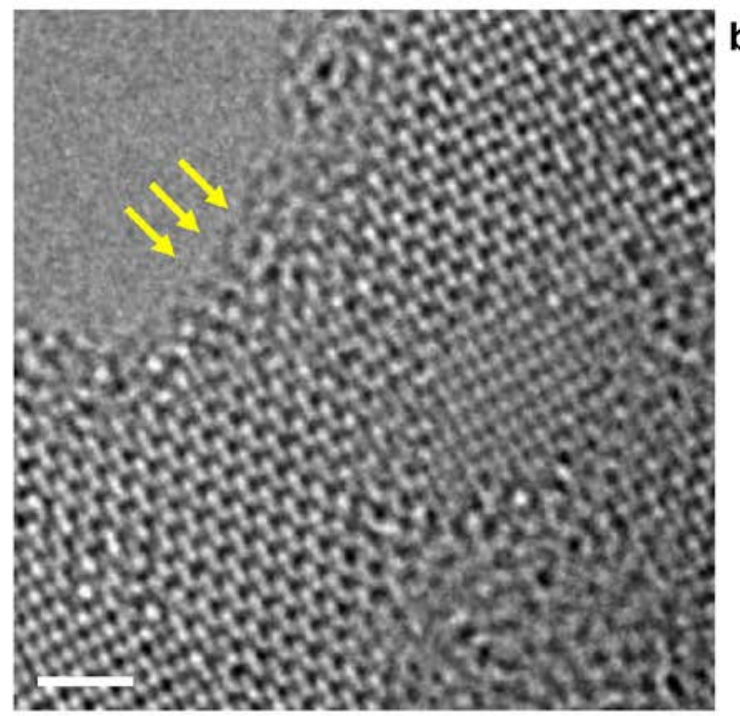

c

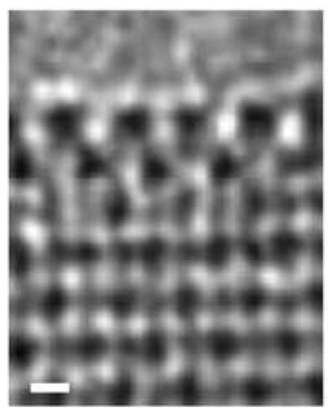

g

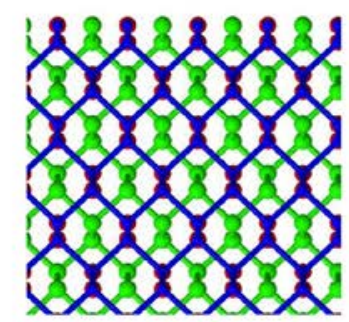

d

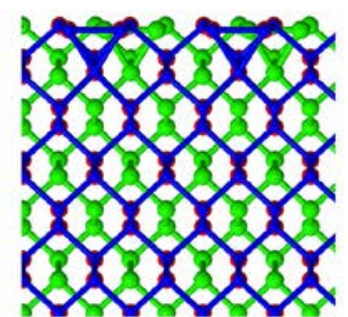

h

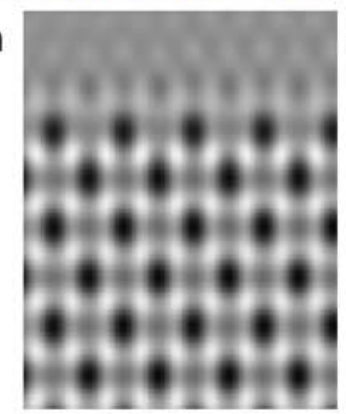

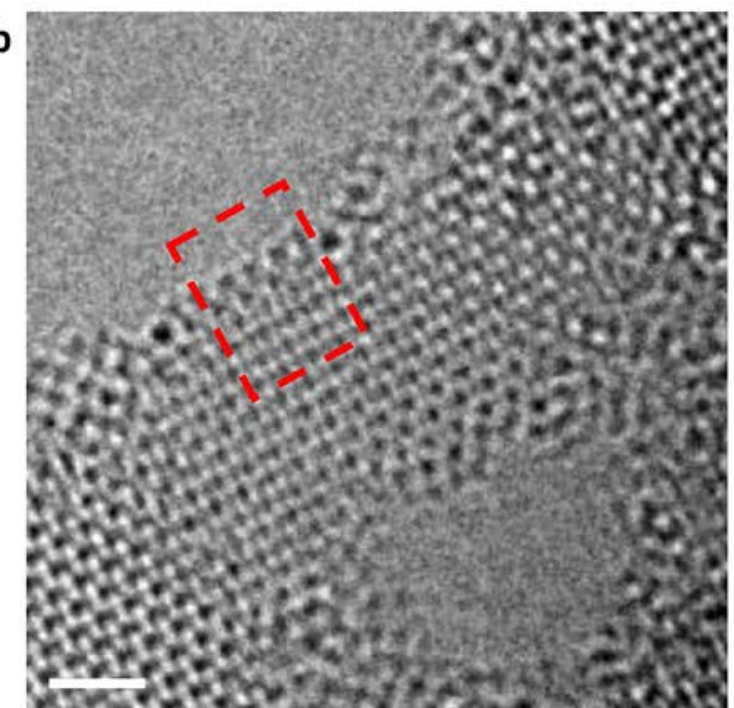

e
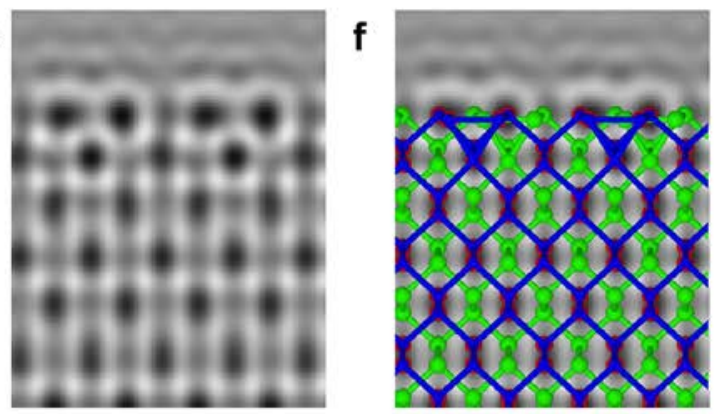

j

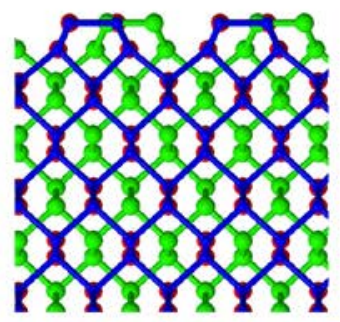

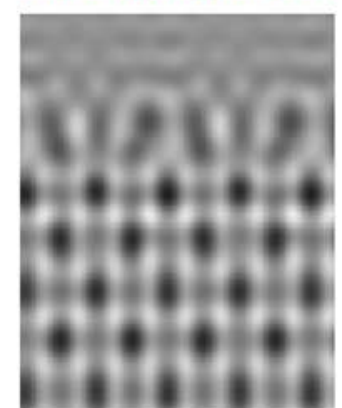

Figure 3.Atomic resolution TEM images of BP edges. (a) TEM image of amorphous structure at BP edge sites as indicated by yellow arrows. Scale bar, 1nm. (b) Crystalline BP edge produced by ebeam irradiation. The image was acquired after $15 \mathrm{sec}$ e-beam irradiation from panel a. Atoms at amorphous edge are etched by e-beam and consequently crystalline edge is exposed. The red box is the field of view for panel c. Scale bar, $1 \mathrm{~nm}$. (c) Zoom-in image of the BP edge. Scale bar, 0.2nm. (d) Atomic model of type-2 reconstructed ZZ edge configuration in triple-layer BP. The atomic layer at the bottom (red) is overlapped with the top layer (blue). (e) TEM image simulation of the atomic model in panel c. Defocus value is -2nm. (f) The same simulation image with atomic model overlay. (g) Atomic model of regular ZZ edge configuration without reconstruction. (h) TEM image simulation of regular ZZ edge structure. Defocus value is -2nm. (i) Atomic model of type-1 reconstructed ZZ configuration of triple layer BP. (j) TEM image simulation of the atomic model in panel i. Defocus value is $-2 \mathrm{~nm}$. 

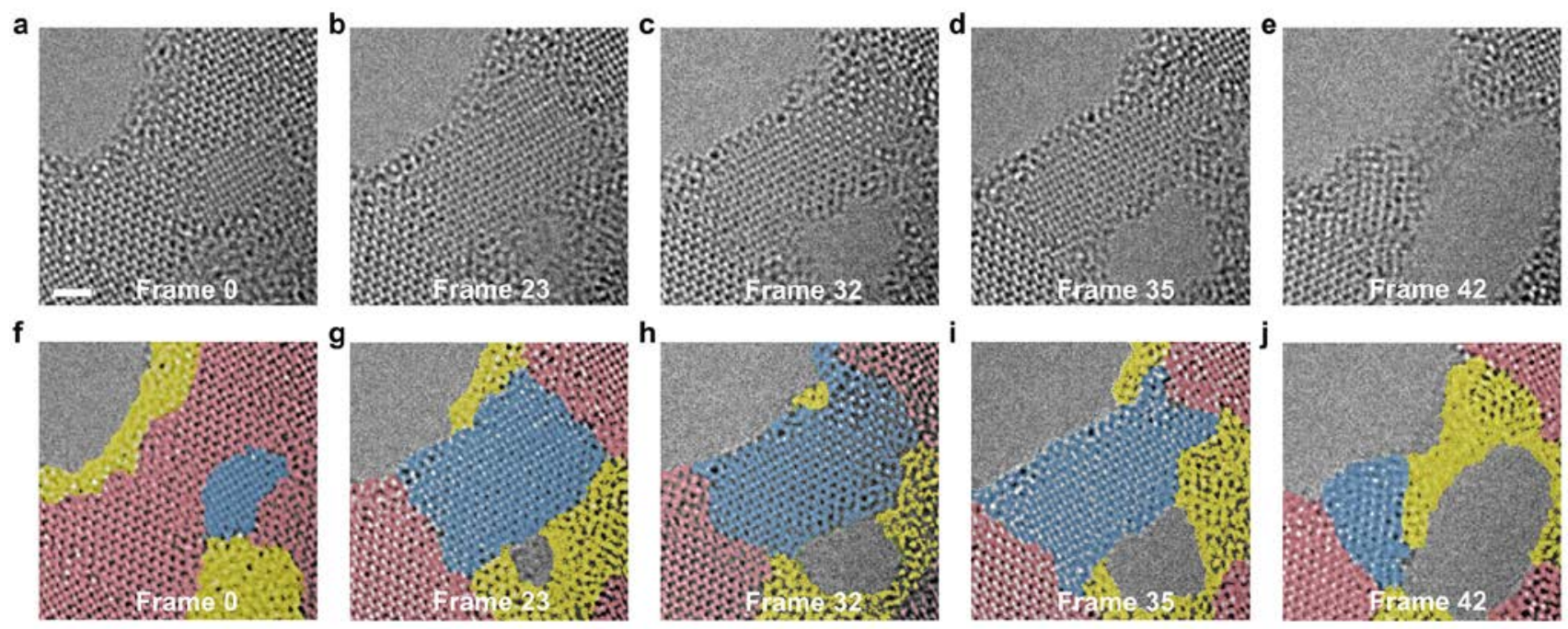

Figure 4. Fabrication of a BP nano-constriction by electron beam. (a-e) A time series of TEM image of BP under electron beam irradiation. Interval time of frame is 0.55 seconds. Scale bar, $1 \mathrm{~nm}$. (f-e) The same TEM images with color overlay. Different colors indicate triple layer (blue), thicker (pink), and amorphous (yellow) regions. 


\section{Reference}

1. Bridgman, P. W. Two new modifications of phosphorus. J. Am. Chem. Soc. 36, 1344-1363, (1914).

2. Hultgren, R., Gingrich, N. S. \& Warren, B. E. The Atomic Distribution in Red and Black P hosphorus and the Crystal Structure of Black Phosphorus. J. Chem. Phys. 3, 351, (1935).

3. Keyes, R. W. The Electrical Properties of Black Phosphorus. Phys. Rev. 92, 580-584, (1953).

4. Ling, X., Wang, H., Huang, S., Xia, F. \& Dresselhaus, M. S. The renaissance of black phos phorus. Proc. Natl. Acad. Sci. U. S. A. 112, 4523-4530, (2015).

5. Li, L. et al. Black phosphorus field-effect transistors. Nature Nanotech. 9, 372-377, (2014).

6. Liu, H. et al. Phosphorene: An Unexplored 2D Semiconductor with a High Hole Mobility. A CS Nano 8, 4033-4041, (2014).

7. Xia, F., Wang, H. \& Jia, Y. Rediscovering black phosphorus as an anisotropic layered mater ial for optoelectronics and electronics. Nature Commun. 5, 4458, (2014).

8. Koenig, S. P., Doganov, R. A., Schmidt, H., Castro Neto, A. H. \& Özyilmaz, B. Electric fie ld effect in ultrathin black phosphorus. Appl. Phys. Lett. 104, 103106, (2014).

9. Castellanos-Gomez, A. et al. Isolation and characterization of few-layer black phosphorus. $2 D$ Mater. 1, 025001, (2014).

10. Li, L. et al. Quantum oscillations in a two-dimensional electron gas in black phosphorus thin films. Nature Nanotech. 10, 608-613, (2015).

11. Wang, X. et al. Highly anisotropic and robust excitons in monolayer black phosphorus. Natu re Nanotech. 10, 517-521, (2015).

12. Youngblood, N., Chen, C., Koester, S. J. \& Li, M. Waveguide-integrated black phosphorus p hotodetector with high responsivity and low dark current. Nature Photon. 9, 247-252, (2015).

13. Yuan, H. et al. Polarization-sensitive broadband photodetector using a black phosphorus verti cal p-n junction. Nature Nanotech. 10, 707-713, (2015).

14. Kim, J. et al. Observation of tunable band gap and anisotropic Dirac semimetal state in blac k phosphorus. Science 349, 723-726, (2015).

15. Das, S. et al. Tunable transport gap in phosphorene. Nano Lett. 14, 5733-5739, (2014).

16. Qiao, J., Kong, X., Hu, Z. X., Yang, F. \& Ji, W. High-mobility transport anisotropy and lin ear dichroism in few-layer black phosphorus. Nature Commun. 5, 4475, (2014).

17. Park, C. M. \& Sohn, H. J. Black Phosphorus and its Composite for Lithium Rechargeable B atteries. Adv. Mater. 19, 2465-2468, (2007).

18. Sun, J. et al. A phosphorene-graphene hybrid material as a high-capacity anode for sodium-i on batteries. Nature Nanotech., advance online publication http://dx.doi.org/10.1038/nnano.2015.1194, (2015).

19. Kang, J. et al. Solvent Exfoliation of Electronic-Grade, Two-Dimensional Black Phosphorus. ACS Nano 9, 3596-3604, (2015).

20. Rodin, A. S., Carvalho, A. \& Castro Neto, A. H. Strain-Induced Gap Modification in Black Phosphorus. Phys. Rev. Lett. 112, 176801, (2014).

21. Fei, R. \& Yang, L. Strain-Engineering the Anisotropic Electrical Conductance of Few-Layer Black Phosphorus. Nano Lett. 14, 2884-2889, (2014).

22. Han, X., Morgan Stewart, H., Shevlin, S. A., Catlow, C. R. A. \& Guo, Z. X. Strain and Or ientation Modulated Bandgaps and Effective Masses of Phosphorene Nanoribbons. Nano Lett. 14, 460 7-4614, (2014).

23. Fei, R. et al. Enhanced Thermoelectric Efficiency via Orthogonal Electrical and Thermal Con ductances in Phosphorene. Nano Lett. 14, 6393-6399, (2014).

24. Jiang, J. W. Thermal conduction in single-layer black phosphorus: highly anisotropic? Nanote chnology 26, 055701, (2015).

25. Jiang, J.-W. \& Park, H. S. Negative poisson’s ratio in single-layer black phosphorus. Nature Commun. 5, 4727, (2014).

26. Zhu, W. et al. Flexible black phosphorus ambipolar transistors, circuits and AM demodulator. Nano Lett. 15, 1883-1890, (2015).

27. Deng, Y. et al. Black Phosphorus-Monolayer $\mathrm{MoS}_{2}$ van der Waals Heterojunction p-n Diode. 
ACS Nano 8, 8292-8299, (2014).

28. Ziletti, A., Carvalho, A., Campbell, D. K., Coker, D. F. \& Castro Neto, A. H. Oxygen Defe cts in Phosphorene. Phys. Rev. Lett. 114, 046801, (2015).

29. Wang, V., Kawazoe, Y. \& Geng, W. T. Native point defects in few-layer phosphorene. Phys. Rev. B 91, 045433, (2015).

30. Tran, V. \& Yang, L. Scaling laws for the band gap and optical response of phosphorene na noribbons. Phys. Rev. B 89, 245407, (2014).

31. Guo, H. Y., Lu, N., Dai, J., Wu, X. J. \& Zeng, X. C. Phosphorene Nanoribbons, Phosphoru s Nanotubes, and van der Waals Multilayers. J. Phys. Chem. C 118, 14051-14059, (2014).

32. Ramasubramaniam, A. \& Muniz, A. R. Ab initio studies of thermodynamic and electronic pr operties of phosphorene nanoribbons. Phys. Rev. B 90, 085424, (2014).

33. Liang, L. et al. Electronic bandgap and edge reconstruction in phosphorene materials. Nano Lett. 14, 6400-6406, (2014).

34. Liu, X. L., Wood, J. D., Chen, K. S., Cho, E. \& Hersam, M. C. In Situ Thermal Decompo sition of Exfoliated Two-Dimensional Black Phosphorus. J. Phys. Chem. Lett. 6, 773-778, (2015).

35. Favron, A. et al. Photooxidation and quantum confinement effects in exfoliated black phosph orus. Nature Mater. 14, 826-832, (2015).

36. Hÿtch, M. J. \& Stobbs, W. M. Quantitative comparison of high resolution TEM images with image simulations. Ultramicroscopy 53, 191-203, (1994).

37. Howie, A. Hunting the Stobbs factor. Ultramicroscopy 98, 73-79, (2004).

38. Meyer, J. C. et al. Direct Imaging of Lattice Atoms and Topological Defects in Graphene M embranes. Nano Lett. 8, 3582-3586, (2008).

39. Girit, Ç. Ö. et al. Graphene at the Edge: Stability and Dynamics. Science 323, 1705-1708, (2009).

40. Meyer, J. C., Girit, C. O., Crommie, M. F. \& Zettl, A. Hydrocarbon lithography on graphen e membranes. Appl. Phys. Lett. 92, 123110, (2008).

41. Lange, S., Schmidt, P. \& Nilges, T. $\mathrm{Au}_{3} \mathrm{SnP}_{7} @$ Black Phosphorus: An Easy Access to Black Phosphorus. Inorg. Chem. 46, 4028-4035, (2007).

42. José, M. S. et al. The SIESTA method for ab initio order- N materials simulation. J. Phys.: Condens. Matter 14, 2745-2779, (2002).

43. Kresse, G. \& Hafner, J. Ab initio molecular dynamics for open-shell transition metals. Phys. Rev. B 48, 13115-13118, (1993).

44. Kresse, G. \& Furthmüller, J. Efficient iterative schemes for ab initio total-energy calculations using a plane-wave basis set. Phys. Rev. B 54, 11169-11186, (1996).

45. Perdew, J. P., Burke, K. \& Ernzerhof, M. Generalized Gradient Approximation Made Simple. Phys. Rev. Lett. 77, 3865-3868, (1996).

46. Jiř́, K., David, R. B. \& Angelos, M. Chemical accuracy for the van der Waals density func tional. J. Phys.: Condens. Matter 22, 022201, (2010).

47. Thonhauser, T. et al. Van der Waals density functional: Self-consistent potential and the natu re of the van der Waals bond. Phys. Rev. B 76, 125112, (2007).

48. Blöchl, P. E. Projector augmented-wave method. Phys. Rev. B 50, 17953-17979, (1994).

49. Kresse, G. \& Joubert, D. From ultrasoft pseudopotentials to the projector augmented-wave $\mathrm{m}$ ethod. Phys. Rev. B 59, 1758-1775, (1999).

50. Troullier, N. \& Martins, J. L. Efficient pseudopotentials for plane-wave calculations. Phys. $R$ ev. B 43, 1993-2006, (1991).

51. Louie, S. G., Froyen, S. \& Cohen, M. L. Nonlinear ionic pseudopotentials in spin-density-fu nctional calculations. Phys. Rev. B 26, 1738-1742, (1982).

52. Monkhorst, H. J. \& Pack, J. D. Special points for Brillouin-zone integrations. Phys. Rev. B 13, 5188-5192, (1976). 


\section{Supporting Information for}

\section{Atomic-scale imaging of few-layer black phosphorus and its reconstructed edge}

Yangjin Lee ${ }^{1}$, Jun-Yeong Yoon ${ }^{1}$, Declan Scullion ${ }^{3}$, Elton J. G. Santos ${ }^{3,4}$, Hu Young Jeong ${ }^{2, *}$, and Kwanpyo $\mathrm{Kim}^{1, *}$

${ }^{1}$ Department of Physics and Applied Mathematics, Ulsan National Institute of Science and Technology (UNIST), Ulsan 689-798, South Korea.

${ }^{2}$ UNIST Central Research Facilities (UCRF), Ulsan National Institute of Science and Technology (UNIST), Ulsan 689-798, South Korea.

${ }^{3}$ School of Mathematics and Physics, Queen's University Belfast, Belfast, BT95AG, United Kingdom

${ }^{4}$ School of Chemistry and Chemical Engineering, Queen's University Belfast, Belfast, BT95AL, United Kingdom

Correspondence to: K.K. (kpkim@unist.ac.kr) or H.Y.J. (hulex@unist.ac.kr)

\section{Table of Contents}

Supplementary Figure S1. TEM analysis of black phosphorus (BP) at extra zone axes.

Supplementary Figure S2. Energy calculations for difference bilayer stacking configurations.

Supplementary Figure S3. Electron diffraction patterns of thin BP flakes.

Supplementary Figure S4. TEM image simulation.

Supplementary Figure S5. Intensity modulation analysis from simulations.

Supplementary Figure S6. TEM images of BP flakes prepared by usual sample preparation process.

Supplementary Figure S7. Edge formation energy calculations for various configurations.

Supplementary Figure S8. TEM image simulation of phosphorene zigzag (ZZ) edge.

Supplementary Figure S9. TEM image simulation of phosphorene zigzag (ZZ) reconstructed edge configuration1.

Supplementary Figure S10. TEM image simulation of phosphorene zigzag (ZZ) reconstructed edge configuration2.

Table S1. Comparison of BP d-spacing with previous literatures.

Table S2. Comparison of intensity ration between measured and simulated images. 
a

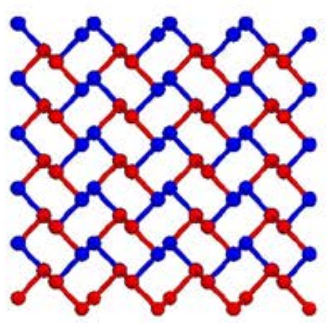

b

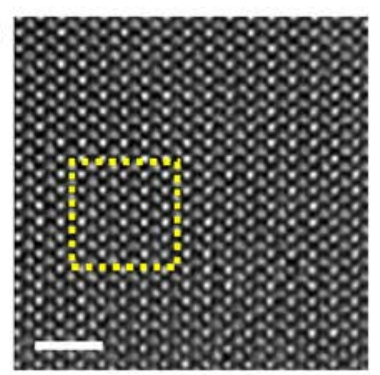

d

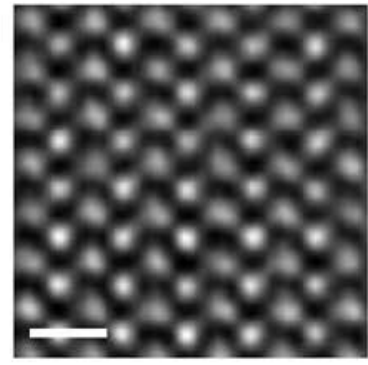

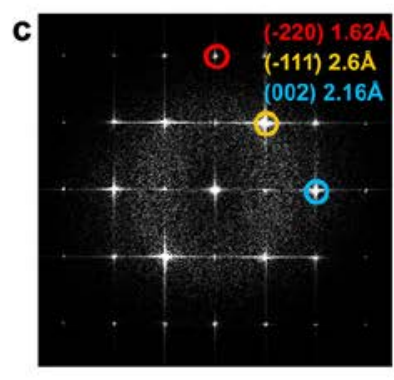

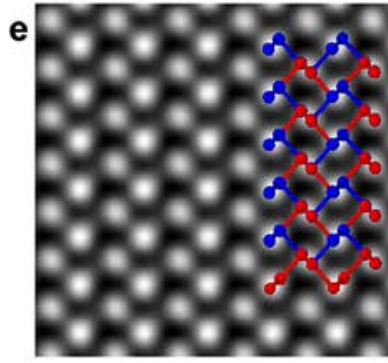

f
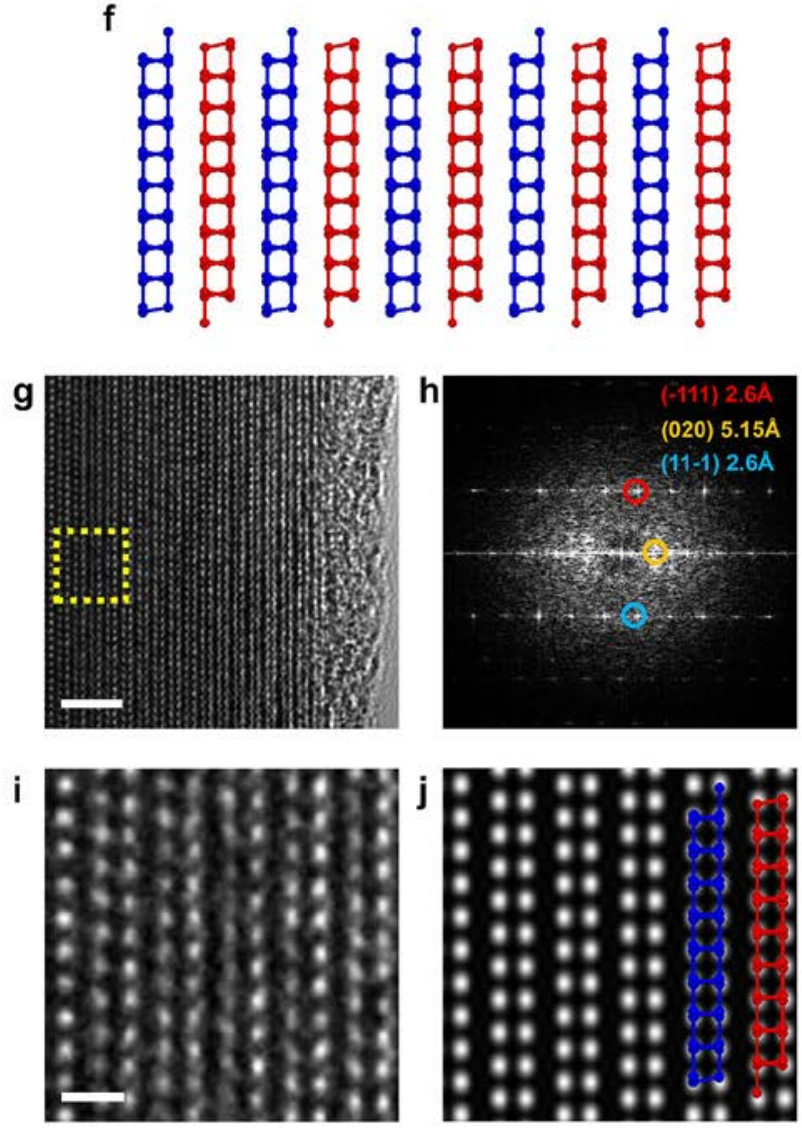

Supplementary Figure S1. TEM analysis of black phosphorus (BP) at extra zone axes. (a) Atomic structural model of BP observed at [110] zone axis. (b) TEM image of BP at [110] zone axis. Scale bar, 2nm. (c) Fourier transform of panel b. The circles indicate low-index diffraction spots. (d) Enlarged view of box in panel b. Scale bar, $0.5 \mathrm{~nm}$. (f) Simulated TEM image from crystal structure shown in panel a. The circles indicate the locations of phosphorus atoms. (f) Atomic model of BP observed at [101] zone axis. (g) TEM image of BP at [101] zone axis. Scale bar, 2nm. (h) Fourier transform of panel g. The circles indicate low-index diffraction spots. (i) Enlarged view of box in panel g. Scale bar, $0.5 \mathrm{~nm}$. (j) Simulated TEM image from crystal structure shown in panel f. The circles indicate the locations of phosphorus atoms. The observed stacking is consistent with $A B$ stacking. 
a
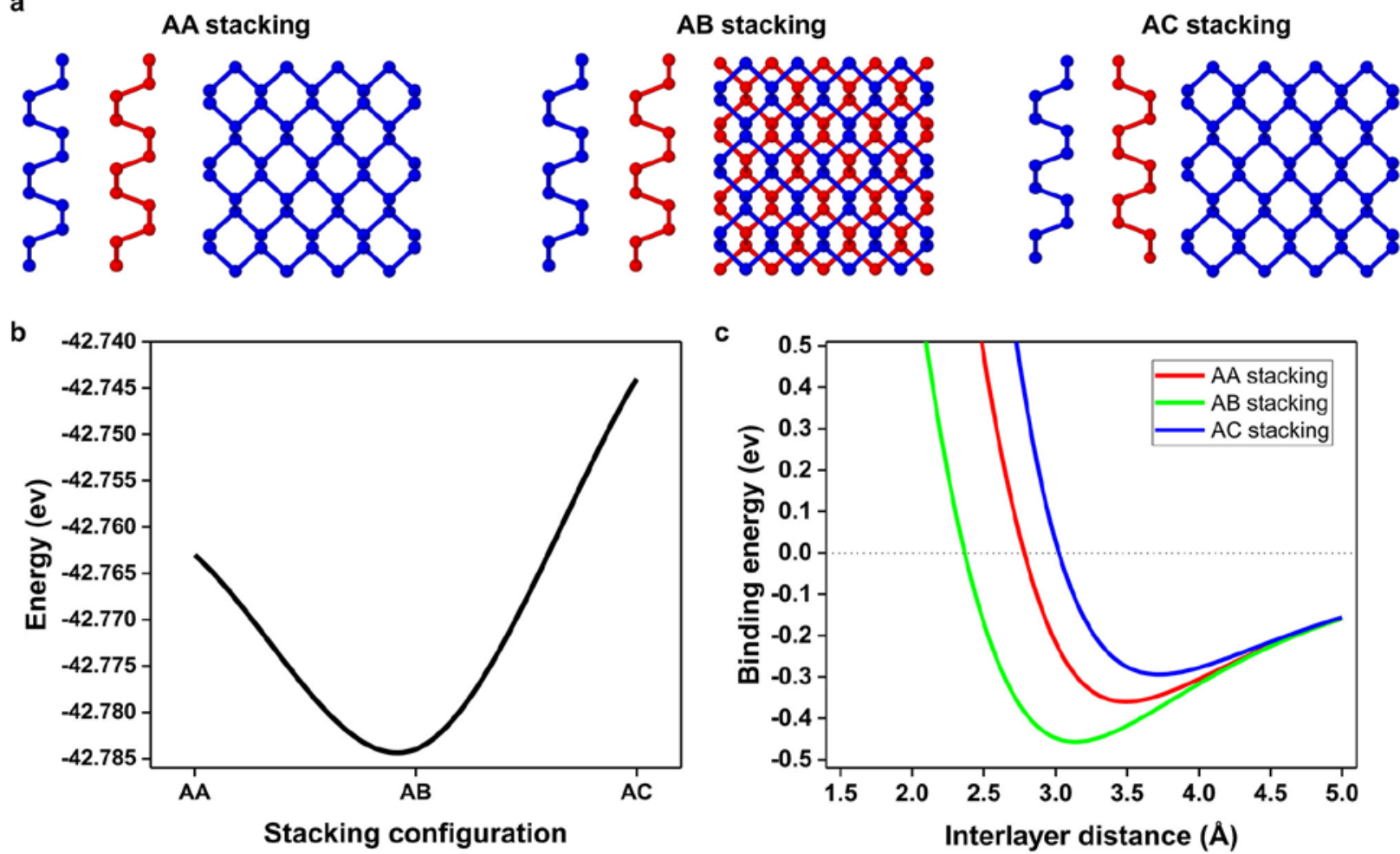

Supplementary Figure S2. Energy calculations for difference bilayer stacking configurations. (a) Bilayer phosphorene model with AA stacking, AB stacking, and AC stacking. (b) Total energy calculations for different stacking configuration. (c) Energy calculation for different stacking as a function of interlayer distance. 

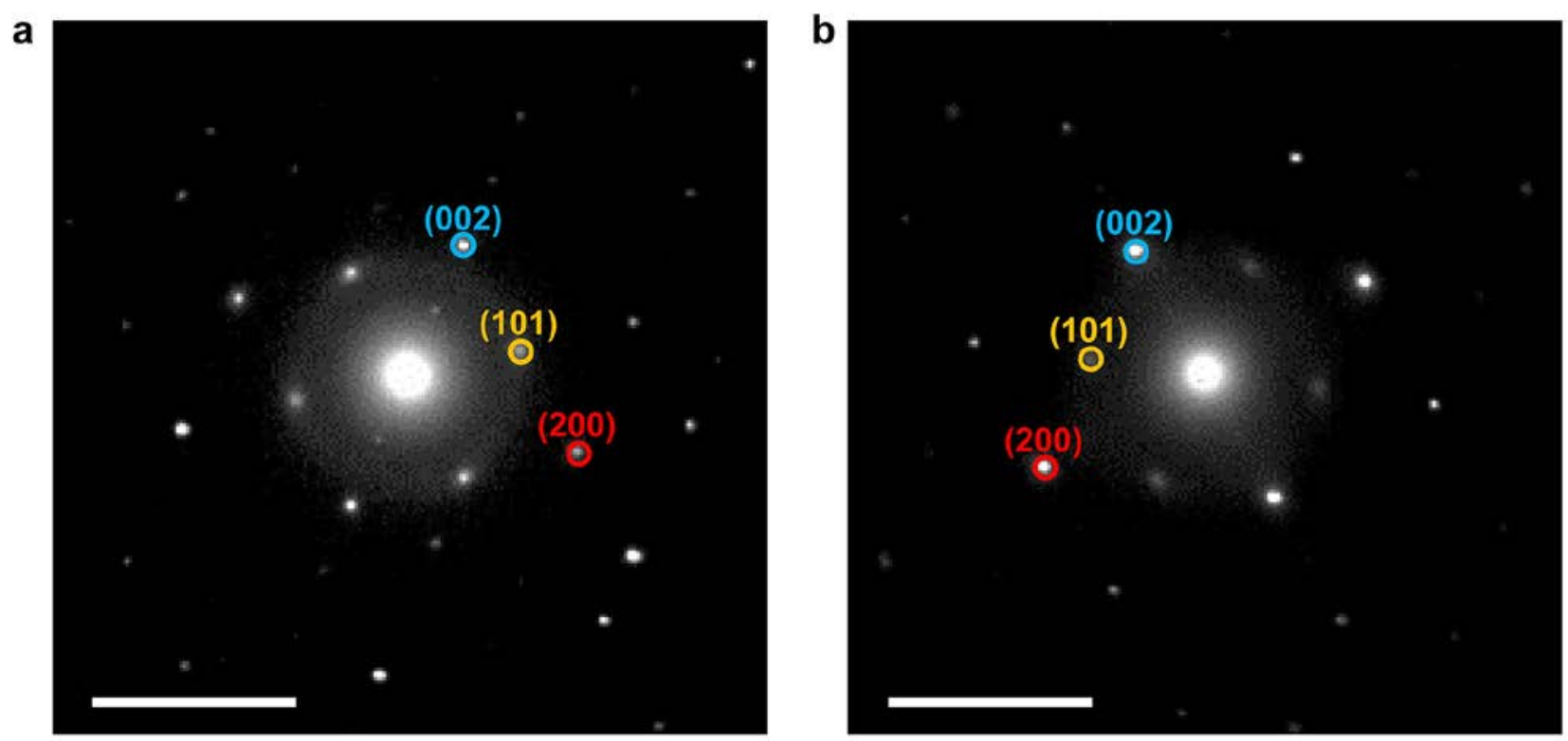

Supplementary Figure S3. Electron diffraction patterns of thin BP flakes. (a) and (b) are acquired with 200nm spot on the thin (3layer) and thick (9layer) region of the flake. Scale bar ; $5 \mathrm{~nm}^{-}$ 1 


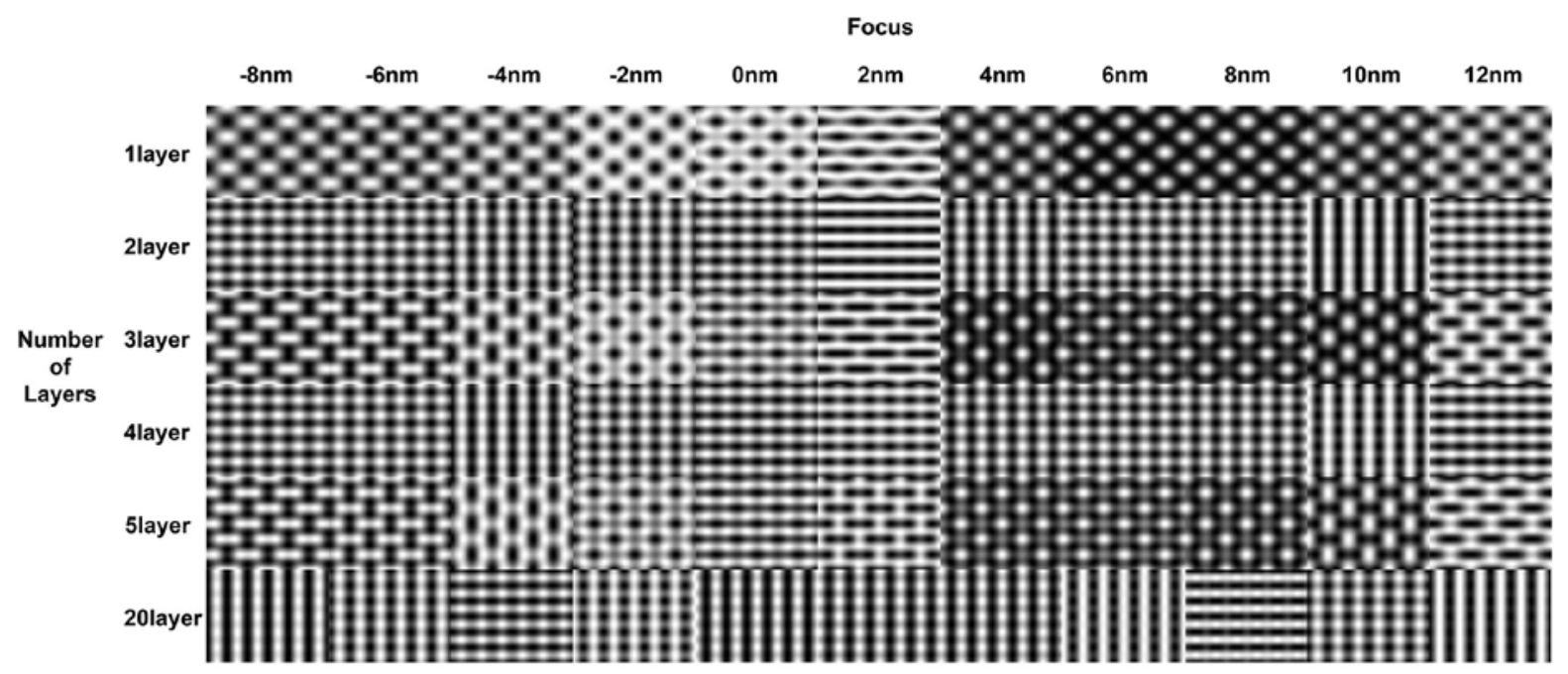

Supplementary Figure S4. TEM image simulation of black phosphorus (BP). Images with various focus conditions (x-axis) and different thickness (y-axis) are shown. Simulated atomic model is the same structure shown in main figure $1 \mathrm{~b}$. 


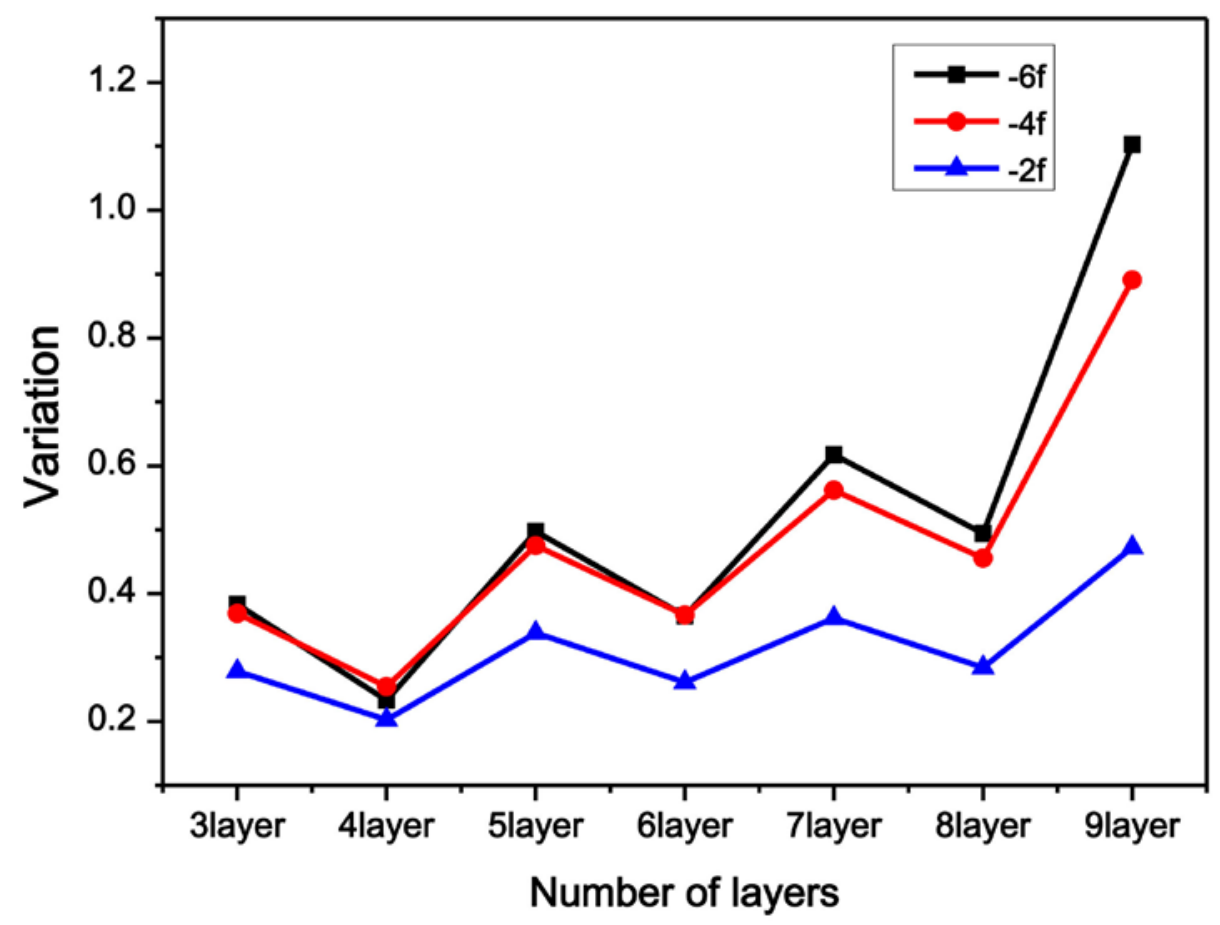

Supplementary Figure S5. Intensity modulation analysis from simulations with different number of layers and various defocus values. 

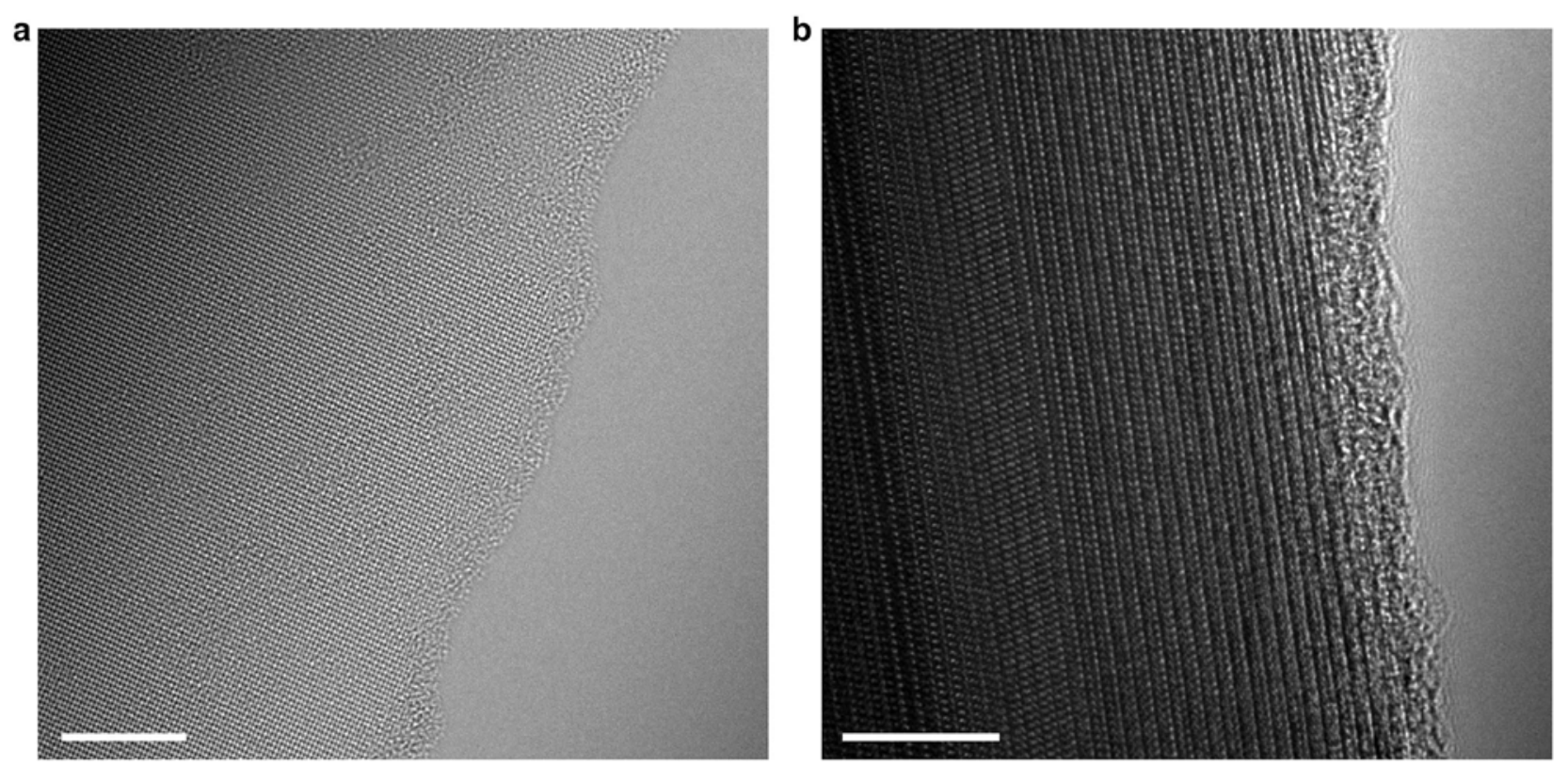

Supplementary Figure S6. TEM images of BP flakes prepared by usual sample preparation process. BP show amorphous regions at edge locations. Scale bar, $5 \mathrm{~nm}$. 
a
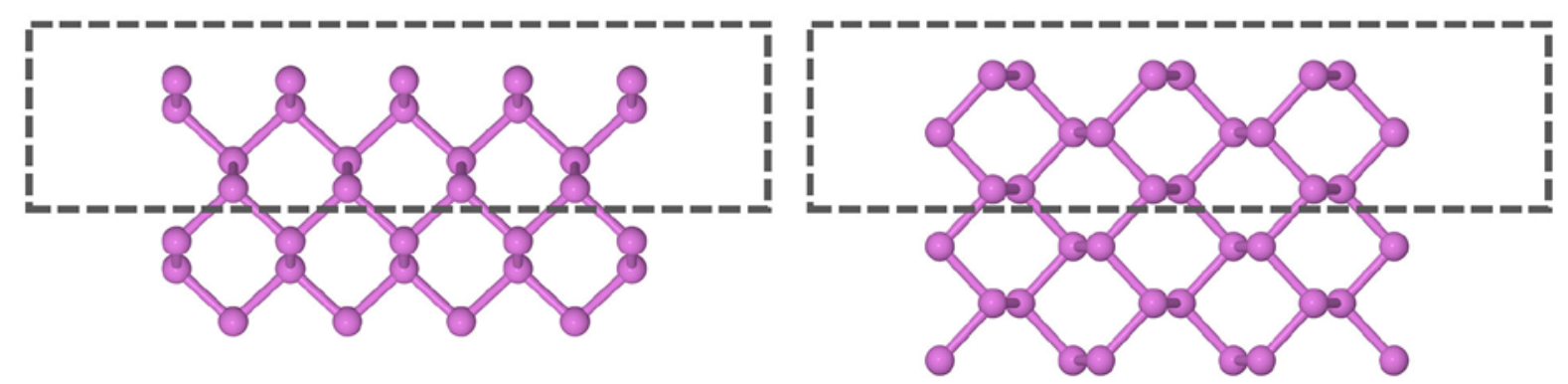

ZZ

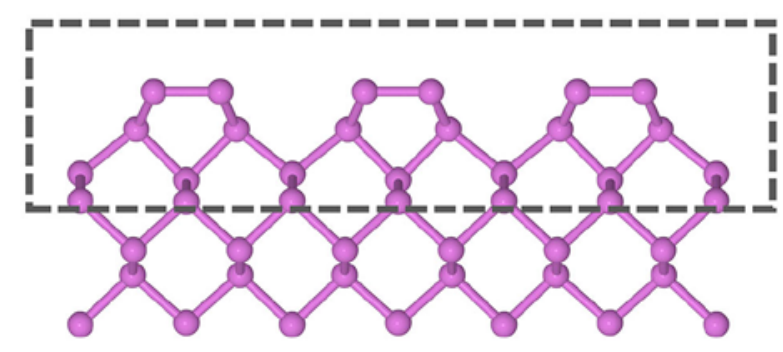

RZZ1
AC

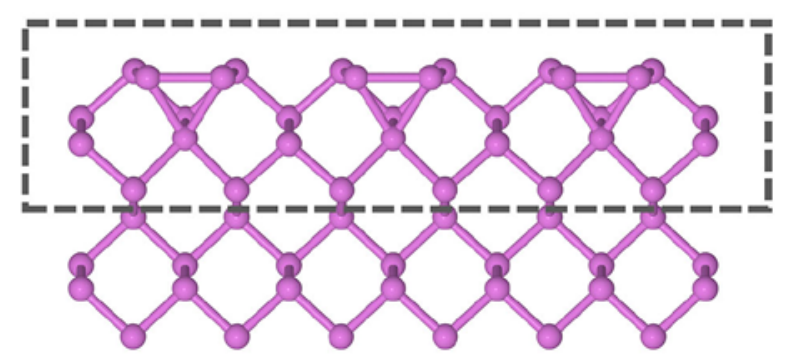

RZZ2

b

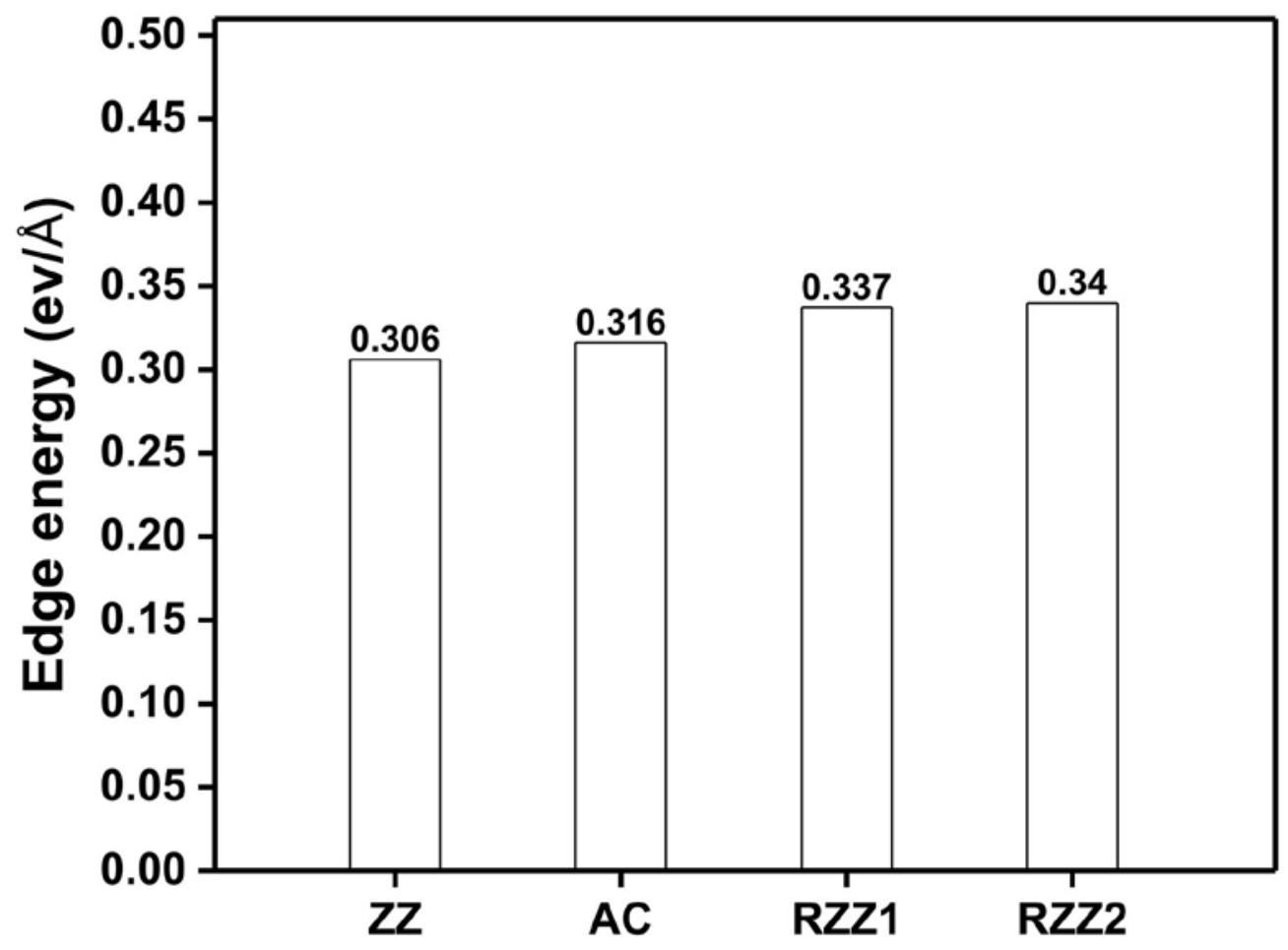

Supplementary Figure S7. Edge formation energy calculations. (a) Atomic models used for edge formation energy calculations. Zigzag (ZZ), armchair (AC), reconstructed zigzag 1 (RZZ1), and reconstructed zigzag 2 (RZZ2) edge configurations are shown. (b) Edge formation energy for different edge configurations. Edge formation energy is given by $E_{\mathrm{f}}=(1 / 2 L)\left(E^{\text {ribb }}-N_{\mathrm{C}} E^{\text {bulk }}\right)$, where $E^{\text {ribb }}$ is the total energy of a ribbon with $N_{\mathrm{C}}$ atoms of the supercell, and $E^{\text {bulk }}$ total energy per atom in monolayer phosphorene. $L$ is the ribbon edge length. 


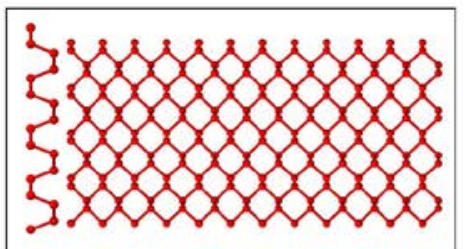

Monolayer

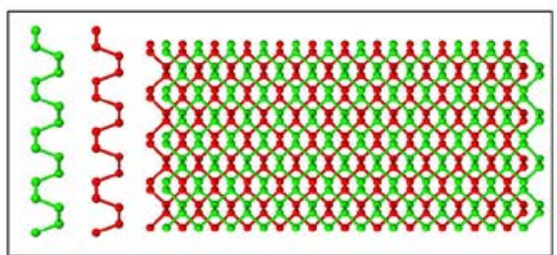

Bilayer

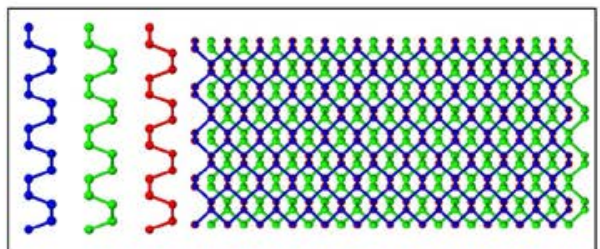

Trilayer

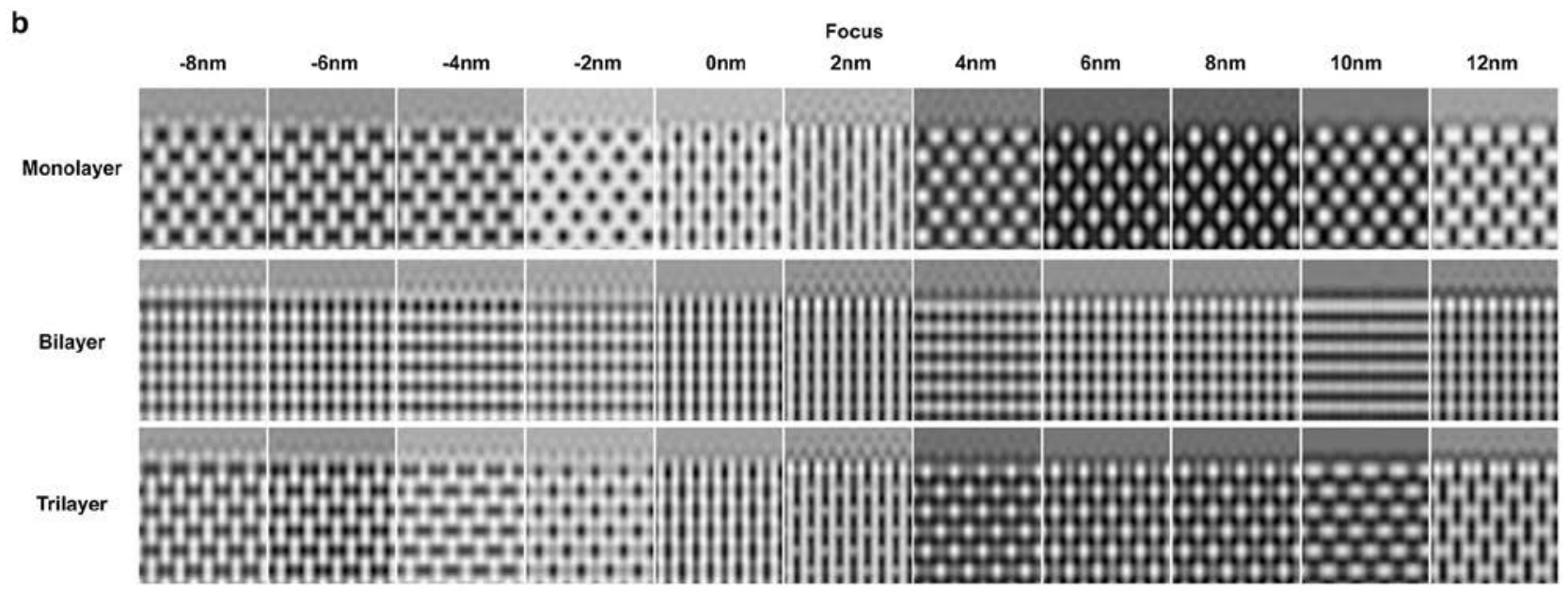

Supplementary Figure S8. TEM image simulation of phosphorene zigzag (ZZ) edge. (a) ZZ edge models used for simulation. (left, side view; right, top view) (b) Simulated TEM images of ZZ edges with various focus (x-axis) and thickness (y-axis). 


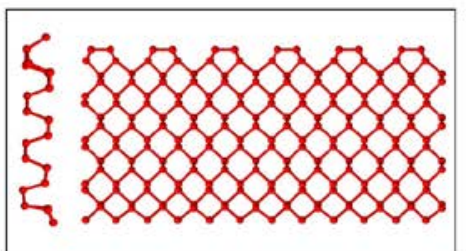

Monolayer
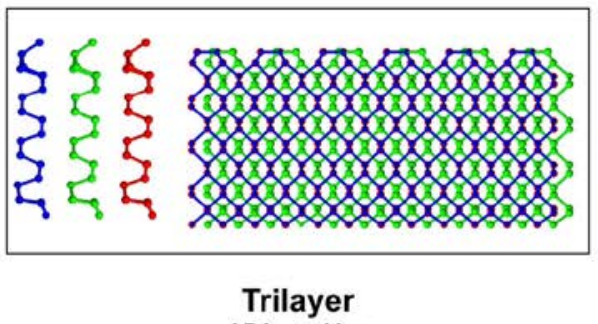

ABA stacking
555

Trilayer

ABA' stacking

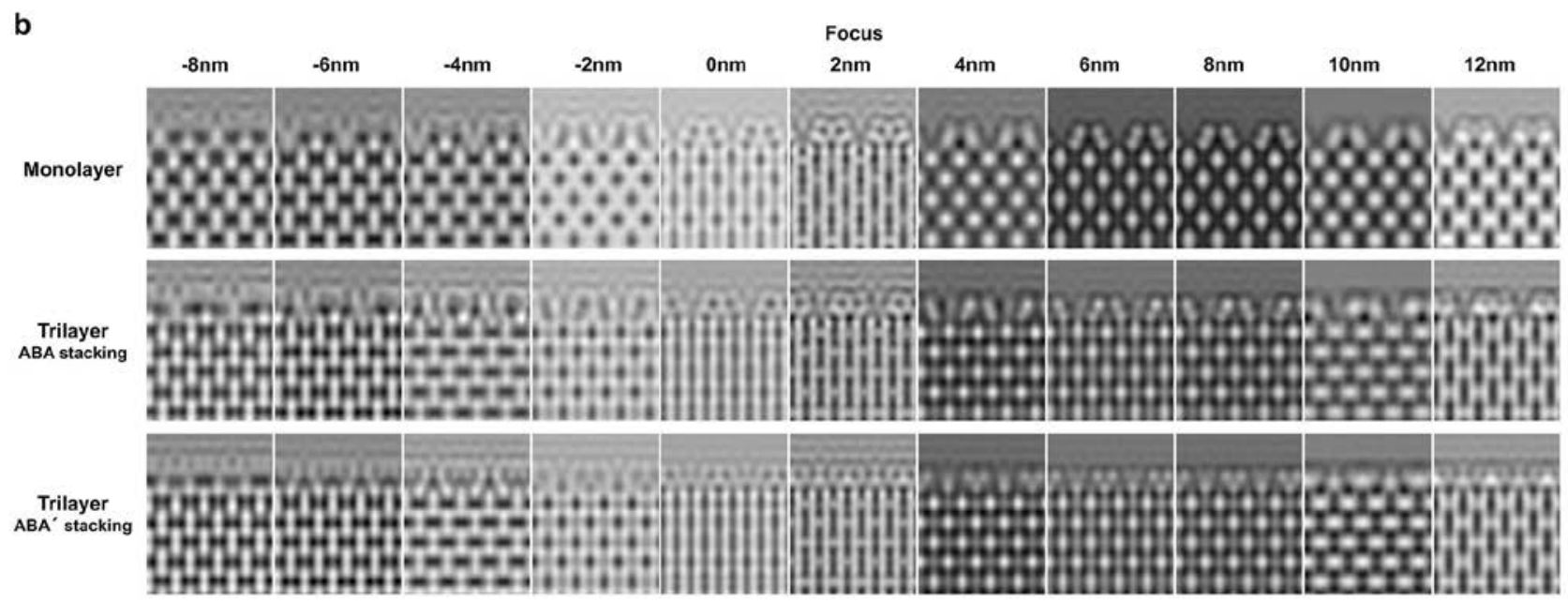

Supplementary Figure S9. TEM image simulation of phosphorene zigzag (ZZ) reconstructed edge configuration1. (a) ZZ reconstructed edge configuration 1 models used for simulation. (left, side view; right, top view) (b) Simulated TEM images of ZZ reconstructed edge configuration 1 models with various focus (x-axis) and thickness (y-axis). 


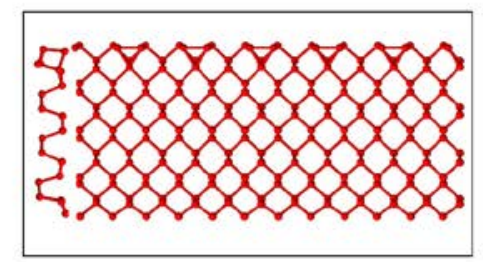

Monolayer

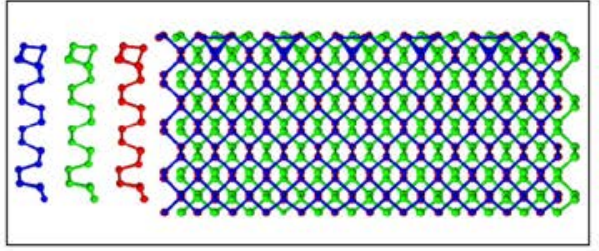

Trilayer

ABA stacking

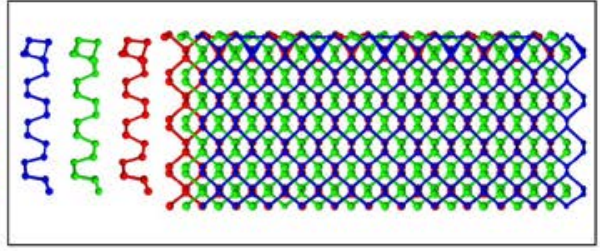

Trilayer

b

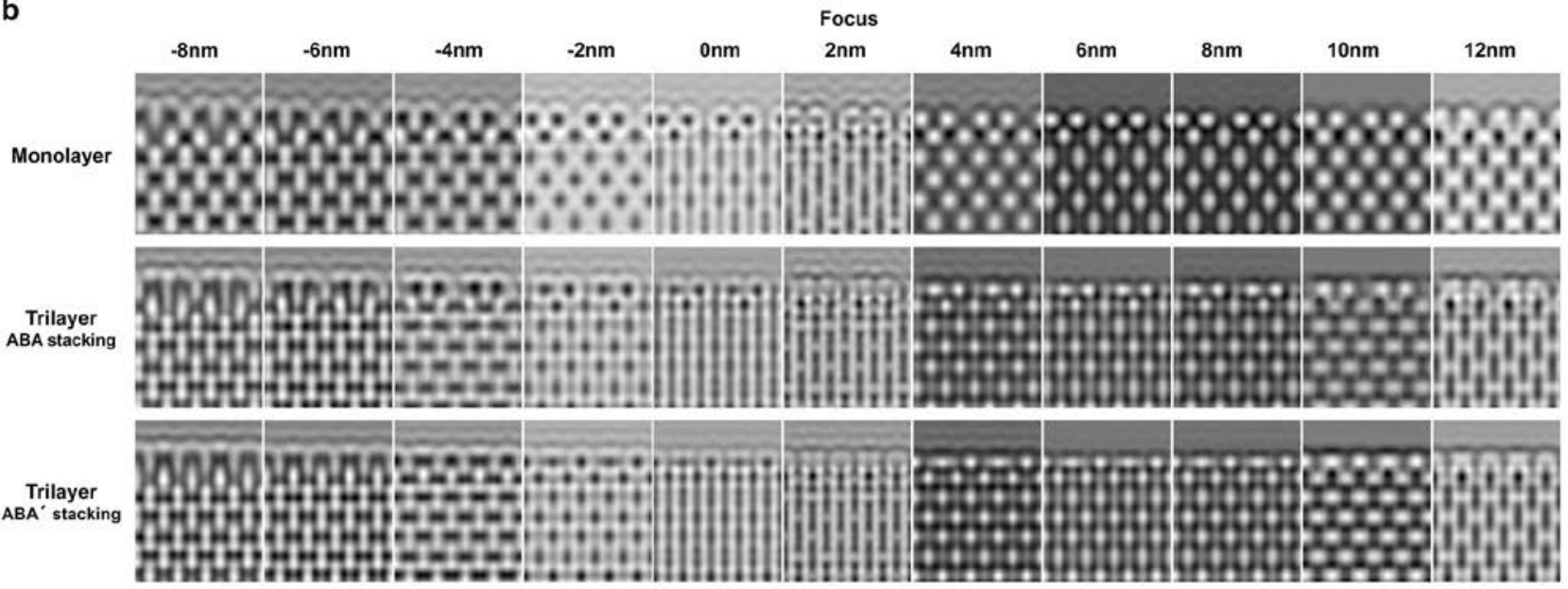

Supplementary Figure S10. TEM image simulation of phosphorene zigzag (ZZ) reconstructed edge configuration2. (a) ZZ reconstructed edge configuration 2 models used for simulation. (left, side view; right, top view) (b) Simulated TEM images of ZZ reconstructed edge configuration 2 models with various focus (x-axis) and thickness (y-axis). 
Table S1. Comparison of BP various d-spacing with previous literatures. (Unit: Å)

\begin{tabular}{ccccccc}
\hline ref & $\mathbf{( 0 0 2 )}$ & $\mathbf{( 2 0 0 )}$ & $\mathbf{( 0 2 0 )}$ & $\mathbf{( 1 1 1 )}$ & $\mathbf{( 0 2 1 )}$ & $\mathbf{( 2 2 0 )}$ \\
\hline In this work & 2.20 & 1.65 & 5.27 & 2.60 & 3.40 & 1.62 \\
2 & 2.19 & 1.640 & 5.24 & 2.56 & 3.36 & 1.579
\end{tabular}


Table S2. Comparison of intensity ratio between measured images and simulated images.

\begin{tabular}{cc|lc}
\hline \hline \multicolumn{2}{c}{ Measured } & \multicolumn{2}{c}{ Simulated } \\
\hline \hline Position & Intensity ratio & Condition & Intensity ratio \\
\hline A & $1.89 \pm 0.12$ & 5L, -6nm focus & 1.78 \\
B & $2.11 \pm 0.14$ & 7L, -6nm focus & 2.21 \\
C & 1.00 & 3L, -2nm focus & 1.00 \\
D & $1.43 \pm 0.22$ & 7L, -15nm focus & 1.39 \\
D' & $1.32 \pm 0.13$ & 7L, -2nm focus & 1.29
\end{tabular}


Supplementary Movie 1. A time series of TEM images of phosphorene nanoribbon formation. The acquisition time of 0.3 second and the processing time of 0.25 second are used. As a result, every image is taken at a rate of frame per 0.55 second during the acquisition. The movie has the frame rate of 4 images/sec. 